\title{
Introdução aos estudos quantitativos utilizados em pesquisas científicas
}

\section{Introduction to quantitative studies used in scientific research}

Júlio Aragão ${ }^{1}$

\section{Resumo}

O presente artigo parte de uma revisão teórica de forma a discutir sobre diferentes tipos de estudos científicos e suas aplicaçốes em diferentes situaçóes de pesquisa. Os principais tipos de estudos quantitativos (relato de caso, série de casos, estudos de incidência, estudos transversais, coortes, caso-controle, estudos ecológicos e ensaios clínicos) são descritos de forma a instrumentalizar a escolha do melhor desenho de um projeto de pesquisa.

Pavavras-chave: Pesquisa, Projetos de Pesquisa, Epidemiologia

\section{Abstract}

This article discusses different types of research design and their applications in different situations from a theoretical review. The main types of quantitative research designs (case report, case series, incidence studies, cross-sectional studies, cohorts, case-control studies, ecological studies and clinical trials) are described to facilitate the choice of the more appropriate study design in a research.

Keywords: Research, Research Design, Epidemiology. 


\section{INTRODUÇÃO}

A realização de uma pesquisa científica se divide em várias etapas, desde o questionamento inicial (hipóteses) até a análise e formulação de conclusôes sobre os dados levantados no estudo. Entre estas, está a escolha do tipo de estudo a ser realizado. Mais do que um fim em si, a escolha ensejada nesta etapa é a de uma ferramenta destinada a uma finalidade específica, em consonância com os objetivos traçados para o estudo.

Embora a abordagem qualitativa em muito tenha avançado nos últimos anos e ganhado espaço cada vez maior no campo científico em nosso país (TURATO, 2005) e em outros, cremos que a abordagem quantitativa não a antagonize, sendo na realidade uma forma complementar e sinérgica de pesquisa (MINAYO \& SANCHES, 1993).

No presente artigo, pretendemos, a partir de uma revisão bibliográfica sobre o assunto, introduzir alguns conceitos relativos aos diferentes tipos de estudos quantitativos disponíveis e sua adequação dentro de diferentes pesquisas e realidades.

\section{ESTUDOS DESCRITIVOS}

Estudos descritivos descrevem a realidade. Não se destinam a explicá-la ou nela intervir. O exemplo mais simples de um estudo descritivo é o relato de caso (ou case). Muito frequente na área de saúde, quando a opção principal do autor é relatar um caso raro ou de evolução incomum, também é freqüente em áreas de gestão ou administração, quando algumas realidades são descritas de forma a partilhar experiências (geralmente, mas não necessariamente positivas). Em algumas situações poder-se-á acumular casos semelhantes, mas igualmente raros na forma de uma série de casos que constituiria assim o nosso segundo tipo de estudo descritivo. A outra forma de estudo descritivo seria o estudo de incidências, que nada mais seria que uma grande tabulação de novos casos ocorridos em uma regiáo em dado período.

Estudos descritivos são fundamentais quando pouco sobre um determinado assunto é conhecido. Relatos de casos e séries de casos vincularam apresentaçôes anômalas do Sarcoma de Kaposi a pacientes homossexuais, levando à pesquisa epidemiológica que descreveria a AIDS (HYMES et al., 1981). Outro importante papel que estudos descritivos podem ter é o de dar conhecimento aos profissionais de uma determinada área ou setor sobre seus dados demográficos (e.g. a taxa natalidade em alunas de uma escola ou mortes por violência doméstica em determinada cidade ou bairro). Estes são os chamados estudos de incidência, que se destinam a descrever incidências de patologias ou situaçóes de interesse durante um período determinado. Estudos que caracterizam demandas e revelam a abrangência de uma pa- tologia em determinada região ou população, como no caso de úlceras de decúbito em idosos (CHAYAMITI \& CALIRI, 2010) ou na deficiência auditiva neonatal (HANNA \& MAIA, 2010) podem demonstrar a importāncia destes dados para os profissionais da assistência e gestores de saúde.

Embora haja uma tendência de se considerar estudos descritivos como estudos "menores" ou de uso restrito, pois deles não se poderia fazer nenhum tipo de inferência, estes estudos podem ser uma ferramenta de gestão muito importante em sistemas de saúde. Um determinado gestor poderá (e muitas vezes o faz) lançar mão de estudos de incidência para caracterizar a demanda de seus serviços, como, por exemplo, sua incidência de procedimentos cirúrgicos ou partos em um hospital. Outro dado que poderá fornecer informaçóes pertinentes é a quantidade de pacientes oriundos de outra área de abrangência. Esse dados são frequentemente tabulados e se transformam em indicadores que compóem uma série histórica.

\section{ESTUDOS ANALÍTICOS I - ESTUDOS OBSER- VACIONAIS}

Estudos analíticos observacionais partem da observação da realidade, sugerindo hipóteses a partir de medidas de associação entre diferentes fatores. Parte-se de uma fator de exposição em busca de uma associaçáo a um evento denominado desfecho. Estudos epidemiológicos não determinam causalidade. Sugerem associação entre fatores e desfechos.

Quatro tipos principais de estudos observacionais podem ser levados a cabo: Estudos tranversais, Estudos de Coorte, Estudos tipo caso-controle e estudos ecologicos.

\section{Estudos transversais}

Estudos transversais ou de corte transversal são estudos que visualizam a situação de uma população em um determinado momento, como instantâneos da realidade (ROUQUAYROL \& ALMEIDA, 2006). Descrevem a situação em uma dado momento e por este motivo frequentemente são classificados de forma errônea como descritivos. $\mathrm{Na}$ realidade, esses estudos possibilitam o primeiro momento de análise de uma associação. Identificados dentro de uma população os desfechos existentes, podemos elencar fatores que podem ou não estar associados a esses desfechos em diferentes graus de associação. Da razão entre as diferentes prevalências entre expostos e náo expostos temos a medida de associação entre ambos, ou Razão de Prevalências (RP). As principais vantagens deste tipo de estudo são o seu baixo custo, sua fácil exequibilidade e a rapidez com que se consegue retorno dos dados obtidos. Sua desvantagem advém 
justamente das restriçóes das análises inferidas (COUTINHO et al., 2008). Estudos transversais são também chamados de inqueritos epidemiológicos e diferem dos estudos descritivos pois destes estudos se pode inferir algo. Voltando ao caso de um gestor de saúde, poderiamos identificar dentre os casos que demandaram prodcedimentos cirurgicos, uma alta taxa de adolescentes ou idosos, por exemplo. Estudos deste tipo revelam a associaçáo entre fatores como obesidade infantil com IMC parental (BERNARDO, 2010) ou de fatores associados à realização de cesarianas (PÁDUA, 2010) são exemplos concretos da utilização destes estudos.

\section{Estudos de Coorte}

Uma coorte nada mais era que uma subdivisão de legionários do exército romano. Da mesma forma, uma coorte epidemiológica reune um grupo de pessoas com as mesmas características para um determinado estudo e seguido por um determinado tempo para que se avalie o desfecho de interesse. Uma vez identificados os portadores do desfecho, estes serão comparados com indivíduos não portadores do desfecho em função da(s) exposiçáo(óes) elencada(s). Identificadas as incidências de casos em expostos e não expostos chegamos ao risco relativo (RR) ou razão de incidências (RUMEL, 1986). Estudos de Coorte são excelentes formas de avaliar associação de fatores e são os mais relevantes dos estudos observacionais frequentemente realizados. Sua grande desvantagem é o custo, visto que muitos indivíduos devem ser monitorados e analizados. Estudos de coorte podem ser realizados tanto com indivíduos sem patologia alguma, como no caso de recém nascidos (GEIB, 2010) quanto em grupos de interesse ou no caso de pacientes portadores de alguma condição específica, como a artrite reumatóide (MOTA, 2010).

\section{Estudos de caso-controle}

A partir da premissa de que em um estudo de Coorte a análise se baseava em comparar as prevalências entre indivíduos com e sem o defecho, alguns epidemiologistas partiram para uma abordagem mais simples mas de eficiência similar. Selecionar um grupo de pessoas com o desfecho em estudo (casos) e compará-los com pessoas sem o desfecho escolhidas aleatoriamente entre a população geral. Esses dois grupos apresentariam taxas diferentes para o fator de exposição em estudo (odds) e a razão entre as diferentes taxas produziria a Razão de chances ou odds ratio (RUMEL, 1986).

Em estudos tipo caso-controle é de primordial importância que tanto casos quanto controles sejam originados na mesma população fonte, sendo necessária especial atenção à seleção dos indivíduos controle. Em trabalho avaliando fatores de risco para queimaduras na infância, WERNECK (1994) optou por utilizar como controles crianças com patologias cirurgicas (abdome agudo e trauma) atendidas no mesmo hospital, pois estes casos eram referenciados ao serviço de cirurgia oriundos da mesma população. Apesar de apresentarem patologias, estas não dividem fatores de risco, possibilitando seu uso como controles para os casos de queimaduras.

\section{Estudos ecológicos}

Estes estudos são similares aos estudos estudos transversais, com a diferença básica de que não medem desfechos em indivíduos e sim em populaçôes. Em um exemplo poderíamos comparar as prevalências de dengue em diferentes cidades em funçãos da área de cobertura com inseticida ou ainda do número de agentes de combate a dengue contratados. O diferencial é que neste estudo cada cidade (ou região, país, grupo etc) é um elemento/sujeito do estudo e não cada indivíduo (CARVALHO \& SOUZA-SANTOS, 2005).

\section{ESTUDOS ANALÍTICOS II - ESTUDOS EXPE- RIMENTAIS}

A diferença nestes estudos reside principalmente no fato de que a intervenção é sempre necessária em estudos experimentais. Ela é o fator a ser estudado e a diferença entre diferentes taxas de um determinado desfecho (desejável ou não) será avaliada e medida de forma a considerar determinada intervenção como recomendável ou não.

O principal tipo de estudo nestes casos é o ensaio clínico. Esse é o tipo de estudo tido como padrão ouro para avaliar se um determinado tratamento ou procedimento/ programa tem efetividade ou não. $\mathrm{O}$ ensaio clínico consiste basicamente em comparar os resultados obtidos por dois grupos de pessoas que receberam determinado procedimento versus indivíduos que não receberam ou que receberam um novo procedimento/tratamento versus indivíduos que receberam o procedimento vigente até o presente momento (NOBRE et al., 2004).

Sua principal desvantagem reside em sua complexidade: geralmente estes estudos requerem equipes envolvidas, profissionais com experiência e recursos financeiros adequados. Procedimentos que evitarão erros metodológicos, tais como a randomização dos indivíduos para determinar quem receberá ou não a intervenção e o vendamento (blind) simples ou duplo, além de equipes de monitorização ou auditoria externa dos dados são apenas alguns dos exemplos que podemos citar. 


\section{CONSIDERAÇÓES FINAIS}

A escolha de uma determinada abordagem na tentativa de prover uma resposta a um dado problema ou hipótese deve levar sempre em conta a realidade do pesquisador, os recursos disponíveis e a aplicabilidade destes resultados no campo prático. Tipos de estudos são abordagens que ensejam operar a conexão entre um modelo ou quadro teórico e a realidade empírica (REICHENHEIM \& MORAES, 1998). Esta conexão, longe de alicerces inquestionáveis garantidos pelo rigor metodológico e científico, repousa em delicados sustentáculos teóricos negociados e assumidos dentro de um contexto global no qual a articulação de diferentes saberes possibilita a construção, ainda que temporária, de um novo conhecimento.

\section{REFERÊNCIAS BIBLIOGRÁFICAS}

1. BERNARDO, C O; FERNANDES, P S; CAMPOS, R M M B; ADAMI, F; VASCONCELOS, F A G . Associação entre o índice de massa corporal de pais e de escolares de 7 a 14 anos de Florianópolis, SC, Brasil. Rev. Bras. Saude Mater. Infant., Recife, v. 10, n. 2, June 2010.

2. CARVALHO, M S; SOUZA-SANTOS, R. Análise de dados espaciais em saúde pública: métodos, problemas, perspectivas. Cad. Saúde Pública, Rio de Janeiro, v. 21, n. 2, Apr. 2005.

3. COUTINHO, L M S; SCAZUFCA, M; MENEZES, P R. Métodos para estimar razão de prevalência em estudos de corte transversal. Rev. Saúde Pública, São Paulo, v. 42, n. 6, Dec. 2008.

4. CHAYAMITI, E M P C; CALIRI, M H L. Úlcera por pressão em pacientes sob assistência domiciliária. Acta paul. enferm., São Paulo, v. 23, n. 1, 2010 .

5. GEIB, L T C; FRÉU C M; BRANDÃO, M; NUNES, M L. Determinantes sociais e biológicos da mortalidade infantil em coorte de base populacional em Passo Fundo, Rio Grande do Sul. Ciênc. saúde coletiva, Rio de Janeiro, v. 15, n. 2, Mar. 2010 .

6. HANNA, K F; MAIA, R A. Triagem auditiva neonatal: incidência de deficiência auditiva neonatal sob a perspectiva da nova legislação paulista. Rev. Bras. Saude Mater. Infant., Recife, v. 10, n. 2, Junho 2010
7. HYMES KB, CHEUNG T, GREENE JB, PROSE NS, MARCUS A, BALLARD H, WILLIAM DC, LAUBENSTEIN LJ. Kaposi's sarcoma in homosexual men-a report of eight cases. Lancet, New York, v. 19 n.2, Sep, 1981

8. MINAYO, M. C. de S.; SANCHES, O.. Quantitativo-qualitativo: oposição ou complementaridade?. Cad. Saúde Pública, Rio de Janeiro, v. 9, n. 3, Sept. 1993

9. MOTA, L M H; LAURINDO, I M M; SANTOS NETO, L L. Características demográficas e clínicas de uma coorte de pacientes com artrite reumatoide inicial. Rev. Bras. Reumatol., São Paulo, v. 50, n. 3, June 2010.

10. NOBRE, M R C; BERNARDO, W M; JATENE, F B. A prática clínica baseada em evidências: Parte III Avaliação crítica das informações de pesquisas clínicas. Rev. Assoc. Med. Bras., São Paulo, v. 50, n. 2, Apr. 2004.

11. PADUA, K S; OSIS, M J D; FAÚNDES, A; BARBOSA, A H; MORAES FILHO, O B . Fatores associados à realização de cesariana em hospitais brasileiros. Rev. Saúde Pública, São Paulo, v. 44, n. 1, fev. 2010 .

12. REICHENHEIM, M. E.; MORAES, C. L.. Alguns pilares para a apreciação da validade de estudos epidemiológicos. Rev. bras. epidemiol., São Paulo, v. 1, n. 2, Aug. 1998 .

13. ROUQUAYROL, M.Z, ALMEIDA FILHO, N.. Epidemiologia e saúde $6^{a}$ edição, Guanabara Koogan , Rio de Janeiro 2006.

14. RUMEL, D. "Odds ratio": algumas consideraçôes. Rev. Saúde Pública, São Paulo, v. 20, n. 3, June 1986.

15. TURATO, E.R. Métodos qualitativos e quantitativos na área da saúde: definiçôes, diferenças e seus objetos de pesquisa. Rev. Saúde Pública, São Paulo, v. 39, n. 3, June 2005.

16. WERNECK, G. L., 1992. Fatores de Risco para Queimaduras Severas na Infância. Tese de Mestrado, Rio de Janeiro: Instituto de Medicina Social, Universidade do Estado do Rio de Janeiro.

\section{Endereço para Correspondência:}

Júlio Aragão - julio.aragao@foa.org.br

Centro Universitário de Volta Redonda

Av. Paulo Erlei Alves Abrantes 1325

Tres Poços - Volta Redonda - RJ - Brasil

CEP: 27240-000 